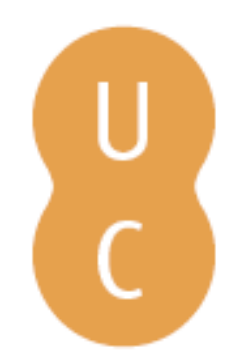

\title{
nombalina
}

\section{Qualidade de vida: versão reduzida (QOL-VR)}

\author{
Autor(es): $\quad$ Cunha, Diana; Relvas, Ana Paula
}

Publicado por: Imprensa da Universidade de Coimbra

URL

persistente: URI:http://hdl.handle.net/10316.2/40588

DOI: $\quad$ DOl:https://doi.org/10.14195/978-989-26-1268-3_4

Accessed : $\quad$ 26-Apr-2023 09:03:28

A navegação consulta e descarregamento dos títulos inseridos nas Bibliotecas Digitais UC Digitalis, UC Pombalina e UC Impactum, pressupõem a aceitação plena e sem reservas dos Termos e Condições de Uso destas Bibliotecas Digitais, disponíveis em https://digitalis.uc.pt/pt-pt/termos.

Conforme exposto nos referidos Termos e Condições de Uso, o descarregamento de títulos de acesso restrito requer uma licença válida de autorização devendo o utilizador aceder ao(s) documento(s) a partir de um endereço de IP da instituição detentora da supramencionada licença.

Ao utilizador é apenas permitido o descarregamento para uso pessoal, pelo que o emprego do(s) título(s) descarregado(s) para outro fim, designadamente comercial, carece de autorização do respetivo autor ou editor da obra.

Na medida em que todas as obras da UC Digitalis se encontram protegidas pelo Código do Direito de Autor e Direitos Conexos e demais legislação aplicável, toda a cópia, parcial ou total, deste documento, nos casos em que é legalmente admitida, deverá conter ou fazer-se acompanhar por este aviso.

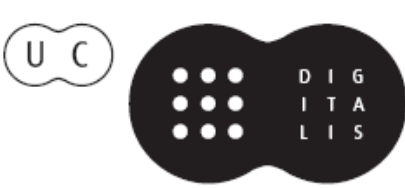


AVALIAÇÃO

$$
\text { FAMILIAR Vorstil Aorracicio }
$$

Q)

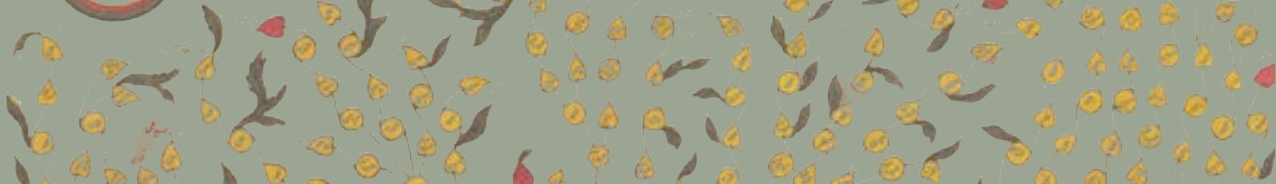

$-1000000$

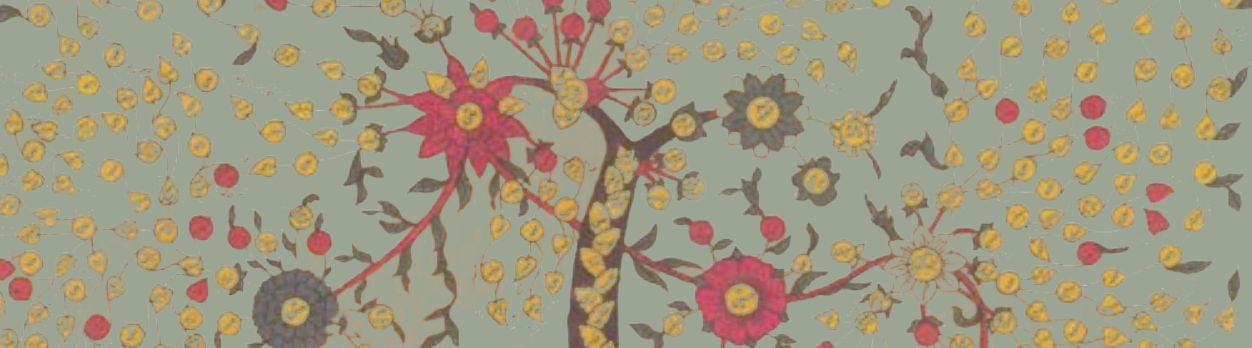

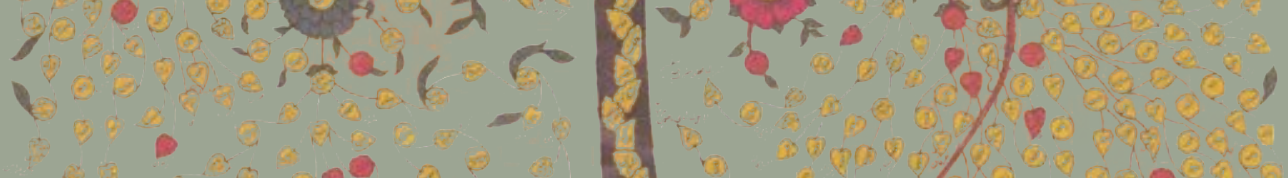

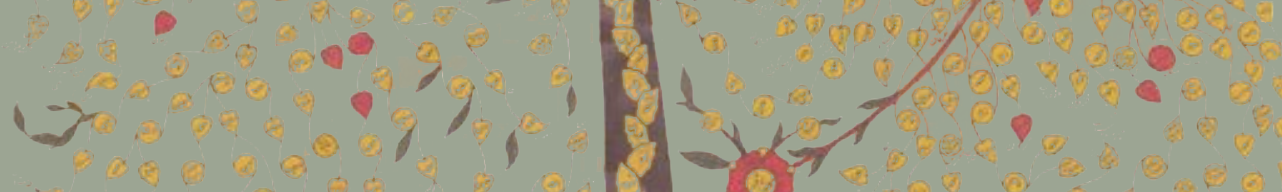

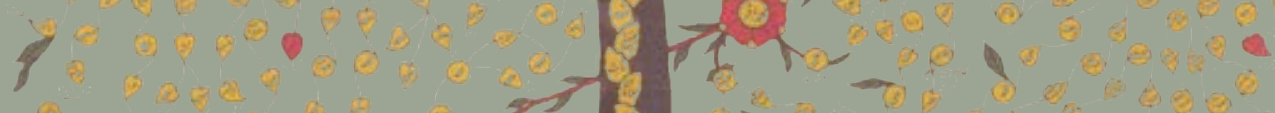

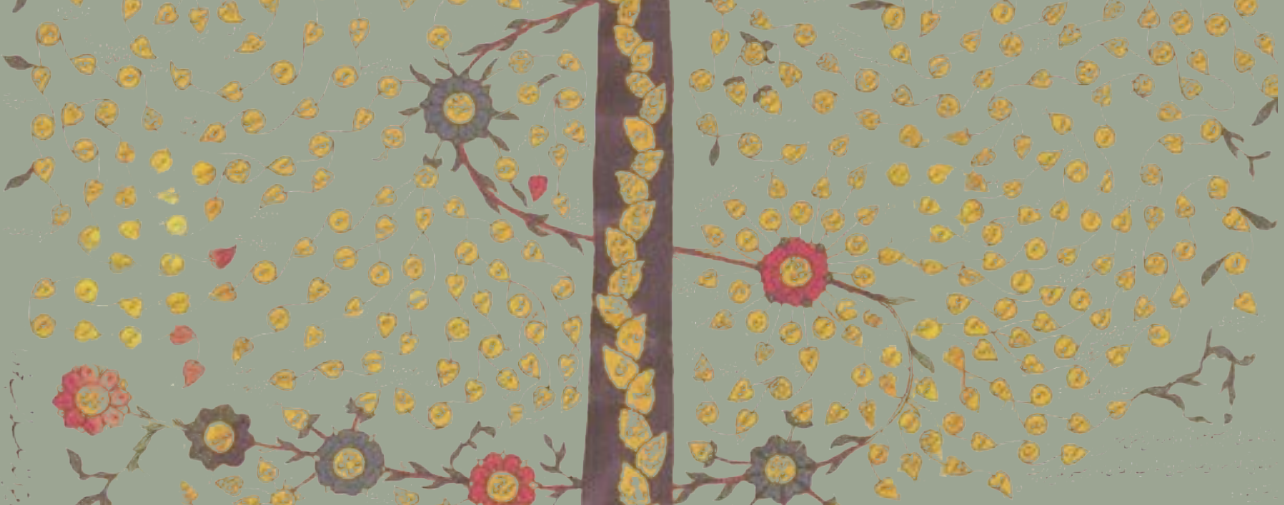

ANA PAULA RELVAS

SOFFA MAJOŔR Wh DE COIMBRA

COORDENAÇÃO 


\title{
QUALIDADE DE VIDA：VERSÃO REDUZIDA ( Q O L - V R )
}

\author{
Diana Cunha \\ Ana Paula Relvas
}

"Quality of life is what a person says it is."

(Twycross, 2003, p. 5)

\section{Resumo}

O Inventário de Qualidade de Vida Familiar (QOL) (Olson \& Barnes, 1982) avalia a satisfação do indivíduo com a qualidade de vida familiar, através de um conjunto de 40 itens. Visando tornar a sua aplicação mais rápida, desenvolveu-se uma versão reduzida (QOL-VR), com 20 itens. Os estudos realizados podem dividir-se em duas fases: 1) redução dos itens e primeiros estudos psicométricos (validade e fiabilidade) $(N=231) ; 2)$ estudos psicométricos (validade e fiabilidade) da versão desenvolvida na etapa anterior numa nova amostra $(N=254)$. Sugere-se uma estrutura de quatro fatores (Bem-Estar Financeiro; Média e Comunidade; Tempo; Família, Amigos e Saúde) que se mostrou ajustada, tanto na fase 1 [AFC $-C F I=.962, G F I=$ .902$, RMSEA: $.050(\mathrm{Lo}=.038, \mathrm{Hi}=.062)]$, como na fase $2[C F I=.946, G F I=$ .904$, RMSEA: .055 (Lo $=.044, \mathrm{Hi}=.065)$ ]. O QOL-VR apresentou razoáveis a bons níveis de consistência interna (escala total e subescalas), em ambas as fases, com valores de alfa de Cronbach a oscilar entre .67 e .89 . O estudo 
apresenta algumas limitações (e.g., amostra não probabilística de conveniência, não estratificada), sugerindo-se a continuidade dos estudos do QOL-VR.

Palavras-Chave: QOL, qualidade de vida familiar, versão reduzida, validade, fiabilidade.

\section{Abstract}

The Inventory of Family Quality of Life (QOL) (Barnes \& Olson, 1982) assesses the satisfaction of the individual with the family quality of life, using a set of 40 items. Aiming to make it application faster, we developed a brief version (QOL-VR), composed by 20 items. This study can be divided into two stages: 1) reduction of items and first psychometric studies (validity and reliability) $(N=231) ; 2)$ psychometric studies (validity and reliability) of the developed version in the previous step, with a new sample $(N=254)$. A four-factor structure is suggested (Financial Wellbeing, Media and Community; Time; Family, Friends and Health) which proved to be adjusted in both phase 1 [AFC - CFI $=.962$, GFI $=.902$, RMSEA: .050 (Lo $=.038, \mathrm{Hi}$ $=.062)]$ and phase $2[\mathrm{AFC}-C F I=.946, G F I=.904$, RMSEA: .055 (Lo $=.044$, $\mathrm{Hi}=.065)]$. The QOL-VR showed reasonable to good internal consistency levels (total and subscales) in both phases, with Cronbach's alpha values ranging between .67 and .89. The study has some limitations (e.g., nonprobabilistic sample of convenience, not stratified), suggesting the continuity of QOL-VR studies.

Keywords: QOL, quality of family life, brief version, validity, reliability.

\section{Instrumento}

O que é, o que avalia e a quem se aplica?

No Quadro 1 encontra-se a ficha técnica relativa ao Qualidade de Vida, Versão Reduzida (QOL-VR; Almeida, 2013) do Quality of Life (QOL) (Olson \& Barnes, 1982). 
Quadro 1.

Ficha técnica do $Q O L-V R$

O que é?

O inventário de Qualidade de Vida apresentado é a versão reduzida (QOL-VR) do Quality of Life (QOL), desenvolvido em 1982, por Olson e Barnes, em St. Paul, Minnesota

O QOL-VR consiste num questionário de auto-resposta, composto por 20 itens que avaliam a qualidade de vida familiar, através da avaliação subjetiva do grau de satisfação com as seguintes áreas de vida: família, amigos, saúde, tempos-livres, média, comunidade, emprego/rendimentos. Os 20 itens encontram-se repartidos por quatro dimensões: Bem-Estar Financeiro; Média e Comunidade; Tempo; e Família, Amigos e Saúde

\begin{tabular}{lcl}
\hline & Estrutura do QOL-VR & \\
\hline Dimensão & Número Itens & Descrição \\
\hline & 5 & $\begin{array}{l}\text { Avalia o grau de } \\
\text { satisfação com o seu } \\
\text { rendimento capacida- } \\
\text { de para fazer face às } \\
\text { despesas familiares e } \\
\text { nével de poupança }\end{array}$ \\
\hline
\end{tabular}

O que avalia?

\begin{tabular}{lcl}
\hline $\begin{array}{l}\text { Média e Comunidade } \\
\text { (MC) }\end{array}$ & 5 & $\begin{array}{l}\text { Avalia o grau de satis- } \\
\text { fação com os média } \\
\text { (e.g., qualidade dos } \\
\text { jornais) e a comunida- } \\
\text { de (e.g., segurança) }\end{array}$ \\
\hline $\begin{array}{l}\text { Tempo } \\
\text { (T) }\end{array}$ & 5 & $\begin{array}{l}\text { Avalia o grau de } \\
\text { satisfação com o tem- } \\
\text { po disponível para a } \\
\text { família, para a lida da } \\
\text { casa e para o próprio }\end{array}$ \\
\hline $\begin{array}{l}\text { Família, Amigos e Saúde } \\
\text { (FAS) }\end{array}$ & $\begin{array}{l}\text { Avalia o grau de } \\
\text { satisfação com a } \\
\text { família, os amigos e } \\
\text { a saúde }\end{array}$ \\
\hline
\end{tabular}

A adaptação da versão portuguesa reduzida do QOL (QOL-VR) atesta a viabilidade da sua aplicação a adultos ( $>18$ anos), da população

A quem se aplica? geral. Note-se que, originalmente, os autores (Olson \& Barnes, 1982) desenvolveram duas formas paralelas do QOL - formulário parental e formulário para adolescentes, encontrando-se o primeiro também adaptado para a população portuguesa (Simões, 2008)

\footnotetext{
Como ter acesso? contactos pessoais e institucionais, bem como dados acerca do propósito da utilização do instrumento (e.g., investigação, prática clínica) e concordar com as condições de utilização e de partilha dos resultados com os autores da versão portuguesa
} 


\section{Fundamentação e bistória}

As primeiras referências à expressão qualidade de vida remontam à Grécia Antiga, onde filósofos como Aristóteles consideravam que "boa vida" designava "a vida que está de acordo com as virtudes, com o bem maior, o bem supremo" (Pimentel, 2006, p. 15). Há, também, indícios de que este termo tenha sido utilizado, pela primeira vez, na literatura médica em 1930 (Seidel \& Zannon, 2004). Mais tarde, em 1964, terá reaparecido com maior intensidade devido a Lyndon Johnson, à data presidente dos Estados Unidos da América, que declarou que a qualidade de vida proporcionada às pessoas seria uma melhor medida do bem-estar da população do que o balanço dos bancos (Fleck et al., 1999).

$O$ que se entende por qualidade de vida tem vindo a alterar-se ao longo dos tempos. Segundo Couvreur (1996), antigamente, falava-se de uma filosofia ou de uma arte para viver enquanto, atualmente considera-se um conceito que invadiu todos os domínios, desde a ecologia à organização do trabalho, passando pela saúde. Talvez se possa afirmar que a década de 90 constitui o principal marco evolutivo do conceito qualidade de vida. Repare-se que foi a partir dessa altura que a ideia de qualidade de vida passou a integrar de forma mais intensa os discursos informais entre as pessoas e os média em geral, assistindo-se, simultaneamente, ao aumento da quantidade e da qualidade de investigações científicas sobre a qualidade de vida e sua relação com questões sociais, culturais e biológicas (Gordia, Quadros, Oliveira, \& Campos, 2011). É também nos anos 90, procurando suprir a confusão conceptual em torno do constructo qualidade de vida (Fleck, 2000) e construir um instrumento de avaliação do mesmo, que a Organização Mundial de Saúde (OMS) reuniu especialistas de várias partes do mundo, formando o grupo de qualidade de vida (grupo WHOQOL). Este grupo desenvolveu uma definição universal, comum a todas as culturas, considerando que qualidade de vida é "a perceção do indivíduo, da sua posição na vida, no contexto da cultura e sistema de valores nos quais ele vive e em relação aos seus objetivos, expetativas, padrões e preocupações” (WHOQOL, 1994, p. 28). 
Esta definição, proposta pela OMS (grupo WHOQOL), também serve de base ao conceito de qualidade de vida familiar, uma vez que o paradigma base é transversal aos dois conceitos, assentando nas mesmas características fundamentais, a multidimensionalidade e a subjetividade (Schalock \& Verdugo, 2006). Multidimensionalidade, uma vez que para avaliar a qualidade de vida é importante ponderar diversos aspetos relativos aos domínios físico, funcional, psicológico e social do indivíduo (Haan, Aaronson, Limburg, Hewer, \& Crevel 1993). E, subjetividade, dado que a sua avaliação resulta da perceção que o indivíduo tem desses domínios (Olson et al., 1983).

Segundo Schalock e Verdugo (2006), a diferença entre qualidade de vida e qualidade de vida familiar reside no foco principal de cada um dos conceitos, ou seja, o primeiro refere-se geralmente ao indivíduo, enquanto o segundo aborda a família enquanto todo. Quando a qualidade de vida é enquadrada por uma perspetiva familiar, foca-se na perceção que o indivíduo tem do seu bem-estar e satisfação com a sua vida familiar em diferentes domínios (Fagulha, Duarte, \& Miranda, 2000). Neste sentido, Olson e Barnes (1982) definem qualidade de vida (familiar) como o sentido de ajustamento da família em relação ao seu meio ambiente. O necessário aumento de complexidade que advém deste tipo de conceptualização de cariz mais familiar acarreta desafios, por exemplo, autores como Park et al. (2003) depararam-se com dificuldades na operacionalização da qualidade de vida familiar. Tal facto deve-se ao elevado número de variáveis que influenciam a vida familiar, nomeadamente: (a) as diferentes perspetivas dos membros da família na definição da sua qualidade de vida; (b) a necessidade de incluir diferentes contextos culturais, sociais e étnicos para garantir uma melhor compreensão da variabilidade existente entre as famílias; e (c) o balanço entre a importância concedida pelas famílias aos diversos indicadores de qualidade de vida e a satisfação ou insatisfação real experimentada pelos seus membros em relação a esses indicadores (Córdoba, Verdugo, \& Benito, 2006).

Apesar disso, David Olson e Howard Barnes (1982) desenvolveram o Quality of Life (QOL). A construção do QOL surge no mesmo âmbito de 
um conjunto de instrumentos de avaliação familiar, entre eles as Family Crisis Oriented Personal Evaluation Scales (F-COPES) (cf. Capítulo 1). Qualquer um desses instrumentos possui como enquadramento teórico de base o Modelo Duplo ABCX de McCubbin e Patterson (1983). Este é uma extensão do Modelo ABCX de Hill (1949), ambos modelos orientados para a compreensão do stress familiar que analisam os acontecimentos indutores de stress que possam afetar a capacidade de adaptação do sistema familiar (Weber, 2011). O modelo ABCX (Hill, 1949) foca-se na capacidade de resposta da família a algo que, de alguma forma, a ameaça e obriga à sua reestruturação, considerando quatro fatores fundamentais, A, B, C e X. Considera-se que A é um evento stressor, B corresponde aos recursos familiares para lidar com as novas exigências, C corresponde à definição que a família faz do acontecimento (individual e coletivamente), e X a crise. O Modelo Duplo ABCX (McCubbin \& Patterson, 1983) complementa o anterior, uma vez que o modelo de Hill (1949) reduz o seu foco às variáveis pré-crise. Assim, adiciona os esforços que os membros da família fazem, ao longo do tempo, para se adaptar, contemplando três fases: a pré-crise, a crise e a pós-crise (McCubbin \& Patterson, 1983). São, assim, acrescentados quatro fatores fundamentais: o fator aA respeitante ao acumular de fatores de stress, o fator bB que se refere aos esforços da família para ativar ou adquirir novos recursos, o fator $\mathrm{cC}$ que considera as modificações da perceção familiar da situação total de crise, e o fator xX que se refere à adaptação da família.

O QOL contempla duas formas paralelas, o formulário parental (40 itens) e o formulário para adolescentes (25 itens), construídos, segundo os autores (Olson \& Barnes, 1982), para que o estudo da qualidade de vida refletisse as preocupações diferenciais de pais e filhos. Trata-se de um instrumento de auto-resposta, cujos itens são cotados através de uma escala de Likert de 5 pontos ( 1 corresponde a "Insatisfeito", 2 a "Pouco Satisfeito", 3 a "Geralmente Satisfeito", 4 a "Muito Satisfeito" e 5 a "Extremamente Satisfeito"). No que respeita à validade destes dois formulários, os autores verificaram (Olson \& Barnes, 1982), através de uma Análise Fatorial Exploratória (AFE) (método de componentes principais), com rotação varimax, a existência de 12 fatores no que respeitava ao 
formulário parental e 11 fatores no formulário para adolescentes. Onze fatores eram comuns a ambos os formulários: Vida Familiar, Amigos, Família Alargada, Saúde, Casa, Educação, Tempo, Religião, Média, BemEstar Financeiro, Vizinhança e Comunidade. Ao formulário parental acrescia o fator Emprego. No que respeita à fiabilidade dos itens do inventário, o formulário parental apresentava um coeficiente alfa de Cronbach de .92 e o formulário para adolescentes de .86, para a escala total (Olson \& Barnes, 1982), considerada uma consistência interna muito boa e boa, respetivamente (Pestana \& Gageiro, 2008).

Em Portugal, o formulário parental é aquele que tem sido estudado e que está na base do QOL-VR.

\section{Estudos em Portugal}

Como foi desenvolvido/ adaptado e validado?

\section{Estudos de tradução e adaptação}

O formulário parental do QOL encontra-se traduzido desde 2006 (tradução do grupo de investigação da Subárea Sistémica, Saúde e Família, da Faculdade de Psicologia e Ciências da Educação da Universidade de Coimbra, coordenado por Ana Paula Relvas) e adaptado e validado para a população portuguesa (Simões, 2008). A versão portuguesa (Simões, 2008) do instrumento apresentou propriedades psicométricas referentes à precisão semelhantes às encontradas pelos autores da versão original, apresentando, para a escala total (40 itens), um coeficiente de alfa de Cronbach de .92, consistência interna considerada muito boa (Pestana \& Gageiro, 2008). No que respeita aos estudos de validade, realizou-se uma AFE (método de componentes principais), seguida de rotação varimax. A versão portuguesa apresenta algumas alterações em relação à versão original, reunindo 11 fatores, em vez dos 12 originais: Bem-Estar Financeiro, Tempo, Vizinhança e Comunidade, Casa, Média, Relações Sociais e Saúde, Emprego, Religião, Família e Conjugalidade, Filhos e 
Educação (Simões, 2008). A principal diferença reside no desaparecimento dos fatores Amigos e Família Alargada, cujos itens foram integrados no fator Relações Sociais e Saúde.

Não obstante a existência desta versão adaptada e validada para a população portuguesa, é reconhecido que as versões reduzidas dos instrumentos facilitam a sua aplicação em termos da gestão do tempo e do cansaço dos participantes (Ballueska \& Gorostiaga, 2012; Podsakoft \& MacKenzie, 1994), representando o seu desenvolvimento uma mais-valia. Para além disso, em 2013, esta necessidade mostrou-se muito relevante, no âmbito de uma investigação de doutoramento conduzida pelas autoras. O protocolo de investigação utilizado era especialmente extenso (cerca de uma hora de preenchimento, em média), possivelmente potenciador de uma menor adesão ao estudo por parte dos participantes, havendo, portanto, necessidade de substituir alguns instrumentos desse protocolo por medidas menos extensas, nomeadamente no que respeitava à avaliação da qualidade de vida. E é neste enquadramento que se iniciam os trabalhos de desenvolvimento e adaptação de uma versão reduzida do QOL (formulário parental) que viria a designar-se QOL-VR.

Os estudos para desenvolver o QOL-VR podem dividir-se em duas fases: 1) redução dos itens e primeiros estudos psicométricos (validade e fiabilidade); 2) estudos psicométricos (validade e fiabilidade) da versão desenvolvida na etapa anterior numa nova amostra da população geral. Uma vez que o protocolo de investigação, a amostra utilizada e as análises estatísticas efetuadas em cada uma destas fases são diferentes, os dados serão apresentados separadamente para cada uma destas fases: Fase 1 e Fase 2.

\section{Fase 1}

Nesta fase utilizaram-se os dados recolhidos através de um questionário de dados sociodemográficos e do formulário parental do QOL (Olson \& Barnes, 1982), tradução portuguesa, realizada em 2006, também utilizada no estudo de adaptação do QOL (Simões, 2008).

Para a realização deste estudo (Fase 1), utilizou-se uma compilação de amostras recolhidas na população geral $(N=231)$ (através do método 
de amostragem por conveniência), entre 2006 e 2008, com os instrumentos referidos, no âmbito dos estudos de Mestrado Integrado em Psicologia Clínica, Subárea Sistémica, Saúde e Família, da Faculdade de Psicologia e Ciências da Educação da Universidade de Coimbra. O nível socioeconómico (NSE) foi calculado segundo a tipologia de Simões (2000) e utilizou-se a tipologia das áreas urbanas do Instituto Nacional de Estatística (INE) (2009) para classificar as áreas de residência - Áreas Predominantemente Urbanas (APU), Áreas Medianamente Urbanas (AMU) e Áreas Predominantemente Rurais (APR). A maioria dos sujeitos são do sexo feminino (66.7\%), a faixa etária mais predominante varia entre os 40 e os 49 anos (35.5\%) e o terceiro ciclo é a escolaridade mais comum (22.1\%) entre os participantes. A maioria é casada (77.5), pertence a um NSE médio (60.2\%) e reside em APU (39.4\%) (cf. Quadro 2).

Quadro 2.

Caracterização da amostra (Fase 1)

\begin{tabular}{llcc}
\hline & & Frequência $(n)$ & Percentagem (\%) \\
\hline \multirow{2}{*}{ Sexo } & Masculino & 77 & 33.3 \\
& Feminino & 154 & 66.7 \\
& $20-29$ & 24 & 10.4 \\
& $30-39$ & 73 & 31.6 \\
& $40-49$ & 82 & 35.5 \\
& $50-59$ & 32 & 13.9 \\
& $60-69$ & 13 & 5.6 \\
& $>70$ & 7 & 3.0 \\
Escolaridade etária & & & \\
& $<1^{\circ}$ Ciclo & 6 & 2.6 \\
& $1^{\circ}$ Ciclo & 31 & 13.4 \\
& $2^{\circ}$ Ciclo & 38 & 16.5 \\
& $3^{\circ}$ Ciclo & 51 & 22.1 \\
& Secundário & 42 & 18.2 \\
Estado civil & Curso profissional & 17 & 7.3 \\
& Licenciatura ou $>$ & 46 & 19.9 \\
& & & \\
& Casado & 179 & 77.5 \\
NSE & União de facto & 52 & 22.5 \\
& & & \\
& Baixo & 68 & 29.4 \\
& Médio & 139 & 60.2 \\
& Elevado & 24 & 10.4 \\
& & & 39.4 \\
& APU & 73 & 31.6 \\
& AMU & 67 & 29.0 \\
\hline
\end{tabular}




\section{Redução dos itens do QOL (Fase 1)}

Após a verificação da adequação da amostra para a realização da análise fatorial - índice Kaiser-Meyer-Olkin (KMO) e teste de esfericidade de Bartlett $-\left(\mathrm{KMO}=.86 ; \chi^{2}(780)=5290.75, p<.001\right)$, realizou-se uma AFE. Para a extração de fatores, utilizou-se o método de componentes principais (CP). Obtiveram-se 11 fatores que explicavam 70.92\% da variância total. De seguida, atendendo ao critério de retenção fatorial, scree plot, proposto por Cattell (1966), realizou-se uma análise fatorial, forçada à extração de quatro fatores, com rotação varimax. O primeiro fator explicava $12.76 \%$ da variância total dos dados, o segundo $12.16 \%$, o terceiro $11.49 \%$ e o quarto 10.57, perfazendo um total de variância explicada de 46.97\% (cf. Quadro 3).

Quadro 3.

Cargas fatoriais (loadings) dos itens do QOL nos fatores (solução após rotação varimax)

\begin{tabular}{|c|c|c|c|c|c|}
\hline \multirow{2}{*}{ Item } & \multicolumn{4}{|c|}{ Fator } & \multirow{2}{*}{$b^{2}$} \\
\hline & 1 & 2 & 3 & 4 & \\
\hline 1. Família & & & & .582 & .793 \\
\hline 2. Casamento & & & & .591 & .714 \\
\hline 3. Filhos & & & & .416 & .690 \\
\hline 4. Crianças & & & & .389 & .572 \\
\hline 5. Amigos & & & & .595 & .589 \\
\hline 6. Familiares & & & & .569 & .592 \\
\hline 7. Saúde & & & & .620 & .719 \\
\hline 8. Saúde familiares & & & & .439 & .570 \\
\hline 9. Condições habitação & .359 & & & .520 & .633 \\
\hline 10. Responsabilidades domésticas & & & .455 & .450 & .765 \\
\hline $\begin{array}{l}\text { 11. Responsabilidades domésticas } \\
\text { outros }\end{array}$ & & & .438 & .458 & .711 \\
\hline 12. Espaço suas necessidades & & & .685 & .329 & .826 \\
\hline 13. Espaço família & & & .640 & .328 & .814 \\
\hline 14. Educação & .467 & & & & .693 \\
\hline 15. Programas educativos & & & .331 & .468 & .728 \\
\hline 16. Tempo-livre & & & .737 & & .816 \\
\hline 17. Tempo si & & & .759 & & .840 \\
\hline 18. Tempo família & & .310 & .703 & & .818 \\
\hline 19. Tempo casa & & & .767 & & .770 \\
\hline 20. Tempo dinheiro & .575 & & .343 & & .629 \\
\hline
\end{tabular}


21. Religião família

22. Religião comunidade

23. Emprego

24. Segurança emprego

25. Televisão

26. Programas televisivos

27. Filmes

28. Jornais e revistas

29. Rendimento

30. Dinheiro necessidades familiares

31. Emergências financeiras

32. Dinheiro deve

33. Poupança

34. Dinheiro futuras necessidades

35. Escolas

36. Compras

37. Segurança

38. Bairro

39. Instalações recreativas

40. Serviços saúde

\% Variância explicada

Nota. Cargas fatoriais (loadings) $\geq .35$ assinaladas a negrito. $N=231$.

\section{.433}

.547

.457

.413

.489

.631

.636

.703

.804

.702

.532

.735

.803
.868

.848

.675

.768

.350

.526

.754

.769

.679

.634

.769

.603

.582

.775

.822

.699

.717

.714

.717

.707

.700

.530

.367

.623

.543

A partir desta base, iniciou-se o trabalho de redução da escala. Para tal, procedeu-se à seleção dos cinco itens de cada fator com maior carga fatorial. Deste processo resultou um conjunto de 20 itens com potencial interesse para a versão reduzida da escala: 1, 2, 5, 6, 7, 12, 16, 17, 18, 19, 27, 28, 29, 30, 31, 33, 34, 35, 36, 37. Estes 20 itens encontram-se distribuídos por quatro fatores: o fator 1 é composto pelos itens 29, 30, 31, 33 e 34 e diz respeito à dimensão Bem-Estar Financeiro; o fator 2 é composto pelos itens 27, 28, 35, 36 e 37 e remete para a dimensão Média e Comunidade; o fator 3 é composto pelos itens 12, 16, 17, 18 e 19 e refere-se à dimensão Tempo; e, por fim, o fator 4 é composto pelos itens 1, 2, 5, 6 e 7 e diz respeito à dimensão Família, Amigos e Saúde (cf. Quadro 4). Posteriormente, realizou-se uma Análise Fatorial Confirmatória (AFC) desta estrutura, apresentada mais adiante. Estes quatro fatores congregam seis dos fatores do instrumento original, tendo os restantes sido eliminados atendendo à análise estatística realizada. 
Quadro 4.

Estrutura fatorial do QOL-VR

\begin{tabular}{ll}
\hline Fator & Itens \\
\hline Família, Amigos e & 1. Família \\
2. Casamento & 5. Amigos \\
& 6. Familiares \\
& 7. Saúde \\
\hline Tempo & 12. Espaço suas necessidades \\
& 16. Tempo livre \\
& 17. Tempo si \\
& 18. Tempo família \\
& 19. Tempo casa \\
\hline Média e Comunidade & 27. Filmes \\
& 28. Jornais e revistas \\
& 35. Escolas \\
& 36. Compras \\
37. Segurança \\
\end{tabular}

A versão reduzida obtida constitui uma versão para adultos e não especificamente parental, uma vez que os itens relativos aos filhos foram eliminados.

Estudos de validade interna: Análise Fatorial Confirmatória (AFC) (Fase 1)

Para obtermos as estatísticas de ajustamento para os resultados da etapa anterior, realizámos uma AFC do modelo resultante: quatro fatores, relacionados entre si - Bem-Estar Financeiro, Tempo, Média e Comunidade e Família, Amigos e Saúde. Este modelo apresentou, em geral, índices de ajustamento adequados $-\chi^{2}=256.154(p<.001), \chi^{2} / d f=1.578$, $C F I=.962, G F I=.902$, RMSEA: $.050(L o=.038, H i=.062)-$ uma vez que $\chi^{2} / d f$ é inferior a 5 , os índices de $C F I$ e $G F I$ são superiores a .90 e RMSEA é inferior a .10 (Marôco, 2010). Para se obter este ajustamento final foram necessárias algumas modificações sugeridas pelos índices 
de modificação. Note-se que apenas se realizaram alterações quando o índice de modificação era elevado e simultaneamente correspondia a uma alteração teoricamente plausível. Por exemplo, acrescentou-se uma correlação entre os erros do item 33 (nível de poupança) e 34 (dinheiro para futuras necessidades da família). Teoricamente, facilmente se aceitam as alterações sugeridas, uma vez que o conteúdo dos itens remete para aspetos que se encontram visivelmente associados (neste caso, poupanças financeiras).

\section{Associação entre subescalas (Fase 1)}

As quatro subescalas encontram-se relacionadas entre si $(p<.05)$, de forma fraca a moderada $(.246<r<.473)$ (Pestana \& Gageiro, 2008). Estas correlações positivas eram esperadas, uma vez que as subescalas representam domínios de um mesmo conceito, a qualidade de vida, tido como multidimensional e dependente não só de fatores pessoais e ambientais como, também, das interações entre os mesmos (Cummins, 2005). Estes dados permitem considerar que o QOL-VR é composto por quatro subescalas dependentes, reforçando a utilidade do seu uso conjunto em detrimento de uma utilização independente.

Estudos de precisão: Análise dos itens, consistência interna (Fase 1)

Os fatores Bem-Estar Financeiro, Tempo e Média e Comunidade apresentam uma consistência interna boa $(.81<\alpha<.89)$ e o fator Família, Amigos e Saúde apresenta uma consistência interna razoável $(\alpha=.72)$ (Pestana \& Gageiro, 2008). A correlação item-total indica uma adequada capacidade discriminante de todos os itens $(>.30)$ (Wilmut, 1975). Este índice de discriminação varia entre .46 e .82 no fator Bem-Estar Financeiro, entre .36 e .83 no fator Tempo, entre .35 e .75 no fator Média e Comunidade e entre .46 e .82 no fator Família, Amigos e Saúde. 
Nesta fase utilizou-se um protocolo de investigação que continha, para além do QOL-VR, um questionário de dados sociodemográficos e duas medidas de validade concorrente: 1) o Systemic Clinical Outcome and Routine Evaluation-15 (SCORE-15) (Stratton, Bland, Janes, \& Lask, 2010; versão portuguesa de Vilaça, Silva, \& Relvas, 2014), um instrumento de auto-resposta que avalia o funcionamento familiar através de 15 itens que se distribuem por três dimensões - Forças da Família, Comunicação Familiar e Dificuldades da Família - e de cinco questões que se reportam à rotina da família, à natureza e impacto dos problemas familiares e possíveis necessidades terapêuticas; o sujeito avalia de que modo é que cada item descreve a sua família, através de uma escala de Likert de 5 pontos (de "Descreve-nos Muito Bem" a "Descreve-nos Muito Mal"), correspondendo uma maior pontuação a maiores dificuldades familiares; e 2) a Escala de Congruência (EC) (Lee, 2002; versão portuguesa de Cunha, Silva, \& Relvas, 2014) que avalia a congruência (estado de harmonia interna e externa, marcado por uma sensação de calma, plenitude, tranquilidade e paz, através do qual o indivíduo pode reagir de forma mais harmoniosa/adaptativa em relação ao seu interior, aos outros e ao contexto)(Banmen, 2002), através de 16 itens, representativos de duas dimensões: Espiritual/Universal e Intra/Interpessoal. O sujeito responde aos itens segundo uma escala de Likert de 7 pontos (de "Discordo Fortemente" a "Concordo Fortemente").

Para se proceder à constituição da amostra, foi considerado o critério de um rácio mínimo (sujeitos/itens) de 5/1 para a realização de análises fatoriais (Wong, Tong, Silva, Abrishami, \& Chung, 2009). Atendendo aos 20 itens do QOL-VR, esta condição impunha um limite mínimo de 100 participantes. No entanto, a amostra recolhida é composta por 254 participantes. Consideraram-se, ainda, os seguintes critérios de inclusão/ exclusão: a) sujeitos com idade igual ou superior a 18 anos, b) nacionalidade portuguesa, e c) saber ler e escrever. A folha de rosto do protocolo de investigação continha a apresentação e os objetivos do estudo, instruções de preenchimento (e.g., solicitação de respostas sinceras e claras; preenchimento integral de todos os instrumentos) 
e esclarecimentos relativos ao caráter confidencial, anónimo e voluntário da participação. Devido a este último facto, os participantes não assinaram qualquer tipo de declaração de consentimento informado (APA, 2010).

A maioria dos participantes $(n=212,83.5 \%)$ foi recrutada através da rede de pessoas conhecidas de um dos autores (método "bola de neve") e cerca de um quinto ( $n=42 ; 16.5 \%)$ a partir de uma recolha on-line. Não se verificaram diferenças estatisticamente significativas entre as duas formas de recrutamento, quer no que respeita ao QOL-VR $[t(248)=$ $0.507, \mathrm{~ns}$ ] como às medidas de validade convergente (SCORE-15 e EC), respetivamente, $t(85)=1.278$, ns e $t(252)=-1.257$, ns.

No caso do recrutamento presencial, esta informação, para além de se encontrar escrita na primeira página do protocolo foi, também, apresentada e discutida com todos os participantes. A administração presencial do protocolo de investigação ocorreu em locais escolhidos pelos participantes (e.g., domicílio, local de trabalho), garantindo-se, dentro do possível e razoável, contextos favoráveis ao preenchimento do protocolo.

O recrutamento dos participantes estendeu-se até ao final do primeiro trimestre de 2012 e deste processo resultou a amostra caracterizada no Quadro 5. Mais uma vez (cf. Fase 1), o NSE foi calculado segundo a tipologia de Simões (2000) e utilizou-se a tipologia das áreas urbanas do INE (2009). A maioria dos sujeitos são do sexo feminino (61.4\%), a faixa etária mais predominante varia entre os 18 e os 25 anos (35.4\%) e a licenciatura (ou superior) é a escolaridade mais comum (37.4\%) entre os participantes. A maioria é solteira (50.0\%), pertence a um NSE médio (60.2\%) e reside em APU (83.0\%) (cf. Quadro 5).

Quadro 5.

Caracterização da amostra (Fase 2)

\begin{tabular}{lccc}
\hline & & Frequência $(n)$ & Percentagem (\%) \\
\hline \multirow{2}{*}{ Sexo } & Masculino & 98 & 38.6 \\
& Feminino & 156 & 61.4 \\
& & & \\
\multirow{3}{*}{ Faixa etária } & $18-25$ & 90 & 35.4 \\
& $26-30$ & 39 & 15.4 \\
& $31-39$ & 38 & 15.0 \\
& $40-49$ & 44 & 17.3 \\
& $50-60$ & 43 & 16.9 \\
\hline
\end{tabular}




\begin{tabular}{lccc}
\hline & $1^{\circ}$ Ciclo & 31 & 12.2 \\
& $2^{\circ}$ Ciclo & 5 & 2.0 \\
$3^{\circ}$ Ciclo & 3 & 1.2 \\
Escolaridade & Secundário incompleto & 34 & 13.4 \\
& Secundário completo & 68 & 26.8 \\
& Curso profissional & 17 & 6.7 \\
& Bacharelato & 1 & 0.4 \\
Licenciatura ou & 95 & 37.4 \\
Estado civil & Solteiro & 127 & 50.0 \\
& Casado & 106 & 41.7 \\
& União de facto & 9 & 3.5 \\
& Divorciado & 8 & 3.1 \\
Viúvo & 4 & 1.6 \\
NSE & Baixo & & 34.3 \\
& Médio & 87 & 60.2 \\
Residência & Elevado & 153 & 5.5 \\
& APU & 211 & 83.0 \\
& APR & 39 & 15.4 \\
& & 4 missings & 1.6 \\
\hline
\end{tabular}

Estudos de validade interna: Análise fatorial confirmatória (AFC) (Fase 2)

O QOL-VR foi testado, desta feita, na nova amostra (amostra fase 2), diferente daquela que lhe deu origem (amostra fase 1). Para tal, realizou-se uma AFC. O QOL-VR, em geral, revelou possuir índices de ajustamento adequados $-\chi^{2}=274.397(p<.001), \chi^{2} / \mathrm{df}=1.759, C F I=.946, G F I=.904$, RMSEA: .055 (Lo = .044, Hi = .065) - uma vez que $\chi^{2} /$ df é inferior a 5 , os índices de CFI e GFI são superiores a .90 e RMSEA é inferior a .10 (cf., Marôco, 2010). Para se obter este ajustamento final, à semelhança do sucedido na fase anterior, também foram necessárias algumas modificações sugeridas pelos índices de modificação. Apenas se realizaram alterações quando o índice de modificação era elevado e simultaneamente correspondia a uma alteração teoricamente aceitável. Por exemplo, acrescentou-se uma correlação entre os erros do item 7 (tempo para si) e 8 (quantidade de tempo livre). Estas alterações fazem sentido do ponto de vista teórico dada a evidente associação/ sobreposição entre o conteúdo dos itens (tempo disponível). 


\section{Associação entre subescalas (Fase 2)}

Também nesta amostra (Fase 2), as quatro subescalas encontram-se relacionadas entre si $(p<.05)$, desta feita de forma moderada $(.325<$ $r<.541$ ) (Pestana \& Gageiro, 2008). Mais uma vez, estas correlações positivas eram esperadas, pelas razões já aduzidas na apresentação dos resultados da fase 1 (Cummins, 2005). Tal como referido anteriormente, o QOL-VR é composto por quatro subescalas dependentes, reforçando a utilidade do seu uso conjunto em detrimento de uma utilização independente.

\section{Estudo de validade convergente (Fase 2)}

Utilizaram-se duas medidas de validade convergente - SCORE-15 e EC. Verificou-se que os quatro fatores do QOL-VR, bem como o resultado total do instrumento, se associam no sentido negativo com o SCORE-15, com uma força moderada (Pestana \& Gageiro, 2008) $(-.505<r<-.308, p$ $<.05$ ). Ou seja, de acordo com o esperado teoricamente (Vilaça, Sousa, Stratton, \& Relvas, 2014), quanto maior a qualidade de vida (familiar), menores as dificuldades familiares e vice-versa.

O fator Família, Amigos e Sáude do QOL-VR, bem como o resultado total do instrumento associam-se, no sentido positivo, à EC. No primeiro caso a associação é moderada $(r=.435, p<.05)$ e no segundo fraca $(r=.297, p<.05)$. Em termos teóricos a direção da relação faz sentido (Lee, 2002), pois quanto maior a qualidade de vida, sobretudo a medida pelo fator Família, Amigos e Sáude, maior a congruência e vice-versa.

Estudos de precisão: Análise dos itens, consistência interna (Fase 2)

Nesta amostra (Fase 2), os fatores Bem-Estar Financeiro e Tempo mantêm uma consistência interna boa (Pestana \& Gageiro, 2008) ( $\alpha=$ $.89, \alpha=.85$, respetivamente). Já os fatores Família, Amigos e Saúde e 
Média e Comunidade diminuem ligeiramente os valores de consistência interna ( $\alpha=.67 ; \alpha=.75$; respetivamente), comparativamente com os apresentados na amostra da Fase 1, apresentando, desta feita, valores razoáveis (Pestana \& Gageiro, 2008). Mais uma vez a correlação item-total indica uma adequada capacidade discriminante de todos os itens (> .30) (Wilmut, 1975). Este índice de discriminação varia entre .58 e .72 no fator Bem-Estar Financeiro, entre .50 e .70 no fator Tempo, entre .46 e .54 no fator Média e Comunidade e entre .48 e .61 no fator Família, Amigos e Saúde.

\section{Aplicação}

\section{Como aplicar, cotar e interpretar?}

O material necessário para a aplicação do QOL-VR é apenas a versão em papel do questionário e uma caneta. A aplicação requer que o sujeito cote cada um dos 20 itens no que diz respeito ao grau de satisfação com os aspetos apresentados. Os itens são cotados numa escala de tipo Likert, de 1 ("Insatisfeito") a 5 ("Extremamente Satisfeito"). O cálculo do resultado total e respetivas subescalas implica a soma dos itens abrangidos.

Os estudos que aqui se apresentam permitiram calcular os primeiros valores de referência. Uma vez que os valores de referência identificados nas duas amostras estudadas (Fase 1 e Fase 2) são muito próximos, optou-se por apresentar, apenas, os verificados na amostra da Fase 2, uma vez que esta foi recolhida mais recentemente e é composta por um número superior de participantes. Assim, apresentam-se no Quadro 6 as médias e desvios-padrão para o resultado total do QOLVR e suas subescalas, para a totalidade da amostra e considerando o sexo dos respondentes.

Passando para a interpretação dos resultados, quer total quer das quatro subescalas, podemos inferir que quanto maiores forem os resultados da escala total e das subescalas maior será a qualidade de vida percebida. 
Quadro 6.

Valores de referência QOL-VR: Amostra total e por sexo

\begin{tabular}{|c|c|c|c|c|c|c|}
\hline \multirow[t]{2}{*}{ Resultados QOL-VR } & \multicolumn{2}{|c|}{$\begin{array}{c}\text { Amostra } \\
\text { Total } \\
(N=254)\end{array}$} & \multicolumn{2}{|c|}{$\begin{array}{l}\text { Sexo Masculino } \\
\quad(n=98)\end{array}$} & \multicolumn{2}{|c|}{$\begin{array}{l}\text { Sexo Feminino } \\
\quad(n=156)\end{array}$} \\
\hline & $M$ & $D P$ & $M$ & $D P$ & $M$ & $D P$ \\
\hline Bem-Estar Financeiro & 13.92 & 4.30 & 14.74 & 4.30 & 13.38 & 4.22 \\
\hline Média e Comunidade & 14.93 & 3.07 & 15.43 & 3.44 & 14.61 & 2.78 \\
\hline Tempo & 15.70 & 4.05 & 16.49 & 3.90 & 15.20 & 4.08 \\
\hline Família, Amigos e Saúde & 18.89 & 3.20 & 19.46 & 2.80 & 18.44 & 3.43 \\
\hline Total & 62.97 & 11.31 & 64.96 & 10.63 & 64.96 & 10.63 \\
\hline
\end{tabular}

\section{Vantagens, limitações e estudos futuros}

A versão reduzida do QOL, o QOL-VR, constitui uma medida de qualidade de vida familiar válida e fiável, enriquecedora do leque de instrumentos de avaliação, disponíveis em Portugal (para a população geral). Para além disso, pelo facto de conter apenas metade dos itens da versão original, economiza tempo na administração, tornando-se menos maçador para os sujeitos. Permite avaliar a qualidade de vida do indivíduo, numa perspetiva familiar e subjetiva, isto é, atendendo ao grau de satisfação percebido pelo sujeito. Uma melhor qualidade de vida familiar associa-se a um funcionamento mais adaptativo das famílias (e dos indivíduos), pelo que a sua avaliação pode ser um importante indicador da saúde das mesmas, permitindo, ainda, a prevenção de eventuais perturbações, físicas ou psicológicas, no sistema familiar (Fagulha et al., 2000).

As limitações deste estudo prendem-se, sobretudo, com as características das amostras (amostras não probabilística de conveniência e não estratificadas e de dimensão relativamente reduzida). Para além disso, o fator 4, Família, Amigos e Saúde, abrange três aspetos relativamente distintos, o que se refletiu na sua menor consistência interna, pelo que a utilização desta subescala deve ser feita cautelosamente. Aliás, recomenda-se, atendendo também à associação entre subescalas, que a 
utilização e interpretação das mesmas comtemple as quatro subescalas e o resultado global.

Futuramente será importante desenvolver estudos que melhorem as características psicométricas do fator 4. Pode igualmente ser útil analisar o desempenho do QOL-VR: a) em outras populações específicas (e.g., doença crónica), e b) em diferentes culturas.

\section{Bibliografia}

Almeida, S. (2013). Escala de Qualidade de Vida Familiar: Desenvolvimento de uma versão reduzida para a população portuguesa (Dissertação de Mestrado Integrado não publicada). Faculdade de Psicologia e de Ciências da Educação da Universidade de Coimbra, Coimbra.

American Psychological Association (2010). Ethical principles of psychologists and code of conduct. Acedido em http://www.apa.org/ethics/code/principles.pdf.

Balluerka, N., \& Gorostiaga, A. (2012). Elaboración de versions reducidas de instrumentos de medida: Una perspectiva práctica. Psychosocial Intervention, 21(1), 103-110. doi: $10.5093 /$ in2012v21n1a7

Banmen, J. (2002). The Satir Model: Yesterday and today. Contemporary Family Therapy: An International Journal, 24, 7-22. doi: 1014365304082

Cattell, R. B. (1966). The screen test for the number of factors. Multivariate Behavioral Research, 1, 245-276.

Córdoba, L. A., Verdugo, M. A., \& Benito, J. G. (2006). Adaptación de la Escala de Calidad de Vida Familiar en Cali (Colombia). In M. Á. Verdugo (Ed.), Cómo mejorar la calidad de vida de las personas con discapacidad. Instrumentos y estratégias de evaluación (pp. 29-41). Salamanca, Espanha: Amarú.

Couvreur, C. (1996). A qualidade de vida: Arte para viver no século XXI. Loures, Portugal: Lusociência.

Cummins, R. A. (2005). Moving from the quality of life concept to a theory. Journal of Intellectual Disability Research, 49, 699-706. doi: 10.1111/j.1365-2788.2005.00738

Cunha, D., Silva, J., Relvas, A. P. (2014). Escala de Congruência (EC). In A. P. Relvas \& S. Major (Coord.) (pp. 113-139), Avaliação familiar: Funcionamento e intervenção (Vol. I). Coimbra: Imprensa da Universidade de Coimbra.

Fagulha, T., Duarte, M. E., \& Miranda, M. J. (2000). A "qualidade de vida": Uma nova dimensão psicológica?. Psychologica, 25, 5-17.

Fleck, M. (2000). O instrumento de avaliação de qualidade de vida da Organização Mundial da Saúde (WHOQOL-100): Características e perspetivas. Ciência e Saúde Coletiva, 5(1), 33-38.

Fleck, M. A., Leal, O. F., Louzada, S., Xavier, M., Chachamovich, E., Vieira, G., \& ... Pinzon, V. (1999). Desenvolvimento da versão em português do instrumento de avaliação de qualidade de vida da OMS (WHOQOL-100). Revista Brasileira de Psiquiatria, 21(1), 19-28. doi: 10.1590/S1516-44461999000100006 
Gordia, A. P., Quadros, T., Oliveira, M., \& Campos, W. (2011). Qualidade de vida: Contexto histórico, definição, avaliação e fatores associados. Revista Brasileira de Qualidade de Vida, 3(1), 40-52.

Haan, R., Aaronson, N., Limburg, M., Hewer, R., \& Crevel, H. (1993). Measuring quality of life in stroke. Stroke, 24, 320-327.

Hill, R. (1949). Families under stress: Adjustment to the crises of war separation and reunion. New York: Harper \& Brothers.

Instituto Nacional de Estatística [Statistics Portugal] (INE). (2009). Tipologia de áreas urbanas. Disponível em http://smi.ine.pt/Versao/Detalhes/1961

Lee, B. (2002). Development of a Congruence Scale based on the Satir Model. Contemporary Family Therapy: An International Journal, 24, 217-239. doi: 1014390009534

Marôco, J. (2010). Análise de equações estruturais. Lisboa: Escolar.

McCubbin, H. I., \& Patterson, J. M. (1983). Family stress process: The Double ABCX Model of family adjustment and adaptation. Marriage and Family Review, 6, 7-37.

Olson, D. H., \& Barnes, H. (1982). Quality of life. In D. Olson et al. (Eds.), Family inventories (pp. 137-148). St-Paul, Minnesota: University of Minnesota, Family Social Science.

Olson, D. H., McCubbin, H. I., Barnes, H., Larsen, A., Mexen, M., \& Wilson, M. (1983). Families: What makes them work. London, England: Sage.

Park, J., Hoffman, L., Marquis, J., Turnbull, A. P., Poston, D., Hannan, H., Wang, M., \& Nelson, L. L. (2003). Toward assessing family outcomes of service delivery: Validation of a quality of life survey. Journal of Intellectual Disability Research, 47, 367-384. doi: $10.1046 / \mathrm{j} .1365-2788.2003 .00497$

Pestana, M. H., \& Gageiro, J. (2008). Análise de dados para ciências sociais: A complementaridade do SPSS ( $5^{\mathrm{a}}$ ed.). Lisboa: Sílabo.

Pimentel, F. (2006). Qualidade de vida e oncologia. Coimbra: Almedina.

Podsakoff, P., \& MacKenzie, S. (1994). An examination of the psychometric properties and nomological validity of some revised and reduced substitutes for leadership scales. Journal of Applied Psychology, 79(5), 702-713. doi: 10.1037/0021-9010.79.5.702

Schalock, R., \& Verdugo, M. A. (2006). Revision actualizada del concepto de Calidad de Vida. In M. Á. Verdugo (Ed.), Cómo mejorar la calidad de vida de las personas con discapacidad. Instrumentos y estratégias de evaluación (pp. 29-41). Salamanca, Espanha: Amarú.

Seidel, E. M. F., \& Zannon, C. M. L. (2004). Quality of life and health: Conceptual and methodological issues. Cadernos de Saúde Pública, 20(2), 580-588.

Simões, J. (2008). Qualidade de vida: Estudo da validação para a população portuguesa. (Dissertação de Mestrado Integrado não publicada). Faculdade de Psicologia e de Ciências da Educação da Universidade de Coimbra, Coimbra.

Simões, M. R. (2000). Investigações no âmbito da aferição nacional do Teste das Matrizes Progressivas Coloridas de Raven (M.P.C.R). Lisboa: Fundação Calouste Gulbenkian/ Fundação para a Ciência e a Tecnologia.

Stratton, P, Bland, J., Janes, E., \& Lask, J. (2010). Developing a practicable outcome measure for systemic family therapy: The SCORE. Journal of Family Therapy, 32, 232-258. doi: $10.1111 / \mathrm{j} .1467-6427.2010 .00507$

Vilaça, M., Silva, J., \& Relvas, A. P. (2014). Systemic Clinical Outcome Routine Evaluation (SCORE-15). In A. P. Relvas \& S. Major (Coord.) (pp. 25-47), Avaliação familiar: Funcionamento e intervenção (Vol. I). Coimbra: Imprensa da Universidade de Coimbra. 
Vilaça, M., de Sousa, B., Stratton, P., \& Relvas, A. P. (2015). The 15-item Systemic Clinical Outcome and Routine Evaluation (SCORE-15) scale: Portuguese validation studies. The Spanish Journal of Psychology, 18, 1-10. doi: 10.1017/sjp.2015.95

WHOQOL Group. (1994). Development of the WHOQOL: Rationale and current status. International Journal of Mental Health, 23(3), 24-56. doi: 10.1080/00207411.1994.11449286

Twycross, R. (2003). Introducing palliative care (4th ed.). Oxford, England: Radcliffe Publishing.

Weber, J. (2011). Individual and family stress and crises. California: SAGE.

Wilmut, J. (1975). Objective test analysis: Some criteria for item selection. Research in Education, 13, 27-56.

Wong, J., Tong, D., Silva, D., Abrishami, A., \& Chung, F. (2009). Development of the functional recovery índex for ambulatory surgery and anestesia. Anesthesiology, 110(3), 596-602. doi: 10.1097/ALN.0b013e318197a16d 